\title{
Sensing Structure based on Surface Plasmonic Resonance in Single Mode Optical Fibers Chemically Etched
}

\author{
L. Coelho* ${ }^{1,2}$, J. M. Almeida ${ }^{1,3}$, J. L. Santos ${ }^{1,2}$, R. A. S. Ferreira ${ }^{4,5}$, P. S. Andre ${ }^{4,6}$, D. Viegas ${ }^{1}$ \\ ${ }^{1}$ INESC TEC (Coordinated by INESC Porto Portugal) Department of Physics, Rua do Campo Alegre 687, \\ 4150-179 Porto, Portugal \\ ${ }^{2}$ Department of Physics, University of Porto, Rua do Campo Alegre, 4150-179 Porto, Portugal \\ ${ }^{3}$ Department of Physics, Universidade de Trás-os-Montes e Alto Douro, 5001-801 Vila Real, Portugal \\ ${ }^{4}$ Department of Physics, University of Aveiro, 3810-193 Aveiro, Portugal \\ ${ }^{5}$ CICECO, University of Aveiro, 3810-193 Aveiro, Portugal \\ ${ }^{6}$ Instituto de Telecomunicações, University of Aveiro, 3810-193 Aveiro, Portugal
}

\begin{abstract}
Many optical systems based on Surface Plasmon Resonance (SPR) have been developed for work as refractometers, chemical sensors or even for measure the thickness of metal and dielectric thin films. These kinds of systems are usually large, expensive and cannot be used for remote sensing. Optical fiber sensors based on SPR has been widely studied for the last 20 years with several configurations mostly using multimode optical fibers with large cores and plastic claddings. Sensors based on SPR present very high sensitivity to refractive index variations when compared to the traditional refractive index sensors. Here we propose a SPR sensor based in a single mode fiber. The fiber end is chemically etched by emersion in a $48 \%$ hydrofluoric acid solution, resulting a single mode fiber with the cladding removed in a small section. A resonance dip around $1580 \mathrm{~nm}$ was attained in good agreement with the simulation scenario that takes into account the real characteristics of the fiber.
\end{abstract}

Keywords: Surface plasmon resonance, optical fiber sensor.

\section{INTRODUCTION}

Surface Plasmon Resonance (SPR) has become very attractive to be used in chemical and bio sensing, usually applying the so-called Kretchmann configuration [1], due to the high sensitivity to the refractive index variations of the surrounding medium. In the past twenty years, much effort was focused on miniaturizing fiber-optic SPR sensors by the application of different techniques. Some examples include distinct coating techniques, R\&D configurations or several types of fibers that could be side-polished, tapered, D-type shape, decladded multimode fibers, using long period gratings, microstructured fibres among other configurations. [2-6]

SPR is a charge-density oscillation that, in certain conditions exists at the interface of two media, metal/ dielectric, with dielectric constants of opposite signs. The charge density oscillation is associated with an electromagnetic wave, and the evanescent field at the interface decays exponentially into both media. This wave is a transverse magnetic polarized one (TM) and its propagation constant at the interface dielectric/metal is given by equation 1 ,

$$
\beta=k \sqrt{\frac{\varepsilon_{m} n_{s}^{2}}{\boldsymbol{\varepsilon}_{m}+n_{s}^{2}}}
$$

where $k$ is the free space wave number, $\varepsilon_{m}$ the dielectric constant of the metal $\left(\varepsilon_{m}=\varepsilon_{m r}+i \varepsilon_{m i}\right)$ and $n_{s}$ the refractive index of the dielectric [7].

*1ccoelho@inescporto.pt;

Fifth European Workshop on Optical Fibre Sensors, edited by Leszek R. Jaroszewicz, Proc. of SPIE Vol. 8794, 87940E · @ 2013 SPIE · CCC code: 0277-786X/13/\$18 · doi: 10.1117/12.2026809 


\section{THE SENSING HEAD}

\subsection{Numerical Simulation}

The numerical simulation was performed in order to obtain the resonance peak at the desired wavelength range for the different layers. It was used the transfer matrix formalism on a system with five layers, core / cladding / metal / dielectric / analyte [8]. This formalism is used to compute the propagation of the radiation through the layer system using the Maxwell's equations subjected to boundary conditions between two adjacent layers. From the basis of this formalism the electromagnetic quantities, electric field vector $\boldsymbol{E}$ and the magnetic field vector $\boldsymbol{H}$, are related by equation 2.

$$
\left[\frac{E_{0}}{H_{0}}\right]=[M]\left[\frac{E_{N}}{H_{N}}\right]
$$

where $[\mathrm{M}]$ is the transfer matrix of the system defined by equation 3 ,

$$
[M]=\left[\begin{array}{ll}
M_{11} & M_{12} \\
M_{21} & M_{22}
\end{array}\right]=\prod_{k=1}^{N-1}\left(\left[\begin{array}{cc}
\cos \delta_{k} & \frac{-i \sin \delta_{k}}{\eta_{k}} \\
-i \eta_{k} \sin \delta_{k} & \cos \delta_{k}
\end{array}\right]\right)
$$

where $\delta_{k}$ is the phase in the $\mathrm{k}^{\text {th }}$ layer given by $\delta_{k}=2 \pi d_{k} \lambda^{-1}\left(\varepsilon_{k}-n_{0}^{2} \sin ^{2} \theta_{0}\right)^{1 / 2}$ and $\eta_{k}$ is the optical admittance given as $\eta_{k}=\varepsilon_{k} /\left(\varepsilon_{k}-n_{0}^{2} \sin ^{2} \theta_{0}\right)^{1 / 2}$. Here $\varepsilon_{k}$ is the dielectric constant and $d_{k}$ is the thickness of the $\mathrm{k}^{\text {th }}$ layer, $\theta_{0}$ is the incident angle, $n_{0}$ is the core refractive index and $\lambda$ the wavelength. The reflectivity coefficient of the multilayer structure is finally given by equation 4 :

$$
R_{p}=\left|r_{p}\right|^{2}=\left|\frac{\left(M_{11}+M_{12} \eta_{k}\right) \eta_{0}-\left(M_{21}+M_{22} \eta_{k}\right)}{\left(M_{11}+M_{12} \eta_{k}\right) \eta_{0}+\left(M_{21}+M_{22} \eta_{k}\right)}\right|^{2}
$$

For gold it were used the tabulated refractive index values with linear interpolation between the data points while for the dielectric experimental values were used [9]. The analyte was assumed to be aqueous with a refractive index around 1.330 RIU. The simulations were performed for different layers thicknesses ( $15 \mathrm{~nm}$ to $25 \mathrm{~nm}$ for gold and $80 \mathrm{~nm}$ to $120 \mathrm{~nm}$ for the dielectric) in order to achieve the optimal configuration.

\subsection{Experimental}

For the sensing device a single mode fiber with a core diameter of $8.6 \mu \mathrm{m}$ and a cladding diameter of $125 \mu \mathrm{m}$ was used. A $10 \mathrm{~mm}$ section of the fiber was chemically etched by immersion in a $48 \%$ hydrofluoric acid solution during 38 minutes at $22^{\circ} \mathrm{C}$ and $60 \%$ of humidity and the etched region became $9 \mu \mathrm{m}$ thick. Instead of the tapering, this technique changes the cladding properties while maintaining the core intact. Due to the vapor acid, transition from the thicker region to the thinner region is not abrupt so the core mode has time to adjust to the new situation without spread out. The new surface created has low roughness and does not need extra treatment.

The metal coating was performed with an electron beam evaporator (model Edwards Auto 306) fitted with a homemade rotary system in order to rotate de fibers with a velocity around 5rpm, producing homogeneous films around the cylindrical fibers. First the etched region was coated with $2 \mathrm{~nm}$ of pure titanium to improve the adhesion of gold. Second a layer with $20 \mathrm{~nm}$ thick film of gold was deposited around the fiber followed by a $104 \mathrm{~nm}$ thick dielectric layer of titanium dioxide. Figure 1 shows a scheme of the fiber with both gold/dielectric layers in the chemical etched region of the fiber. The image on the right is a picture of the produced sensor. It is possible to see the smooth transition from the thick section to the thin section.

The dielectric layer was produced by injecting pure oxygen in the electron beam vacuum chamber with a stable pressure around $3.1 \times 10^{-3} \mathrm{mbar}$ to induce titanium oxidation during the deposition, with a rate of $0.04 \mathrm{~nm} / \mathrm{s}$. The refractive index 
values of this last layer were measured in an AutoSE spectroscopic ellipsometer (HORIBA Scientific) in the 400-800 nm spectral region. From these data, a refractive value index value of $2.2 \mathrm{RIU}$ at $1550 \mathrm{~nm}$ wavelength was estimated.

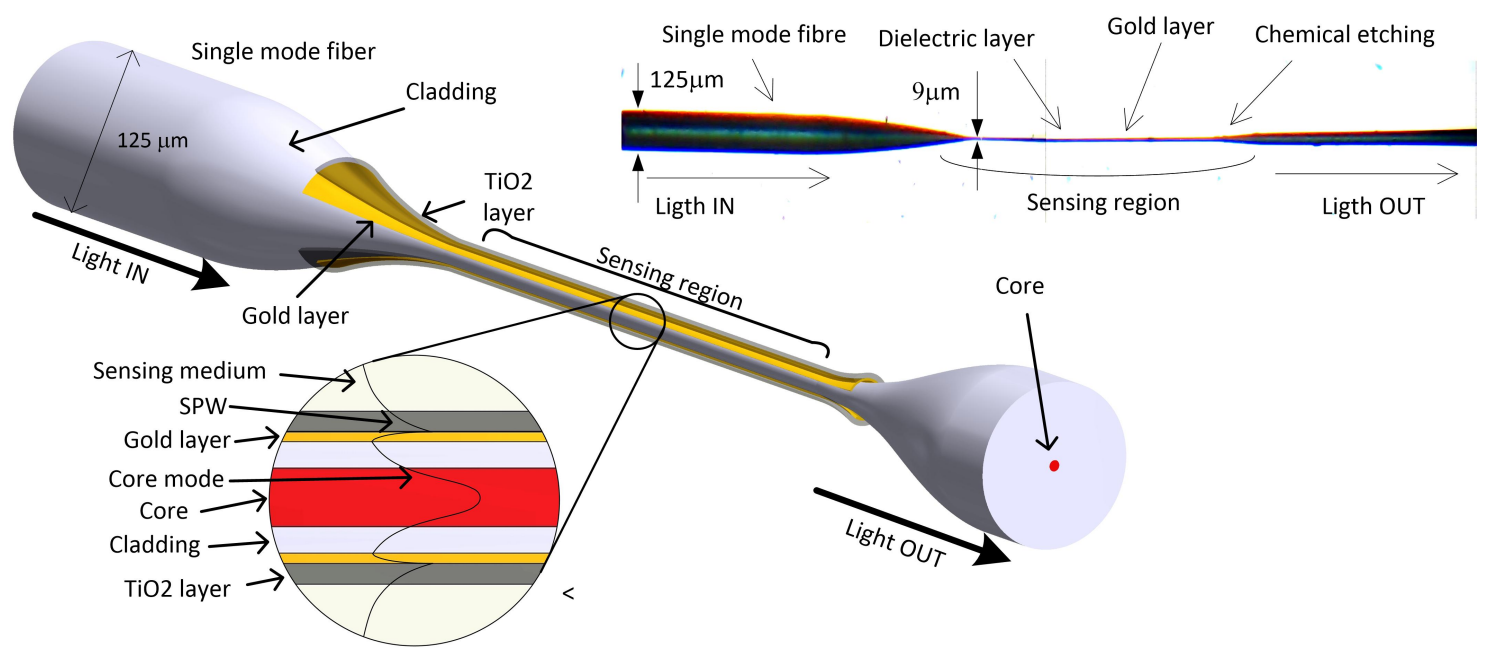

Figure 1.Schemes of (left) the fiber with the gold and dielectric layers in the chemical etched region and (right) of the sensor.

The spectrum was obtained in transmission mode operation with a spectral analyzer from FiberSensing (model FS2200SA) with a resolution of $2.5 \mathrm{pm}$.

\section{DISCUSSION AND RESULTS}

Figure 2 presents the resonant dip from the SPR sensor obtained by simulation. The refractive index of the surrounding medium was changed from 1.325 RIU to 1.335 RIU and the spectral response can be observed in Figure 2-a) were the resonant dip shifts to higher wavelengths as the refractive index values increase. A sensitivity of $4857 \mathrm{~nm} / \mathrm{RIU}$ is theoretically reached with this sensing head, Figure 2-b).

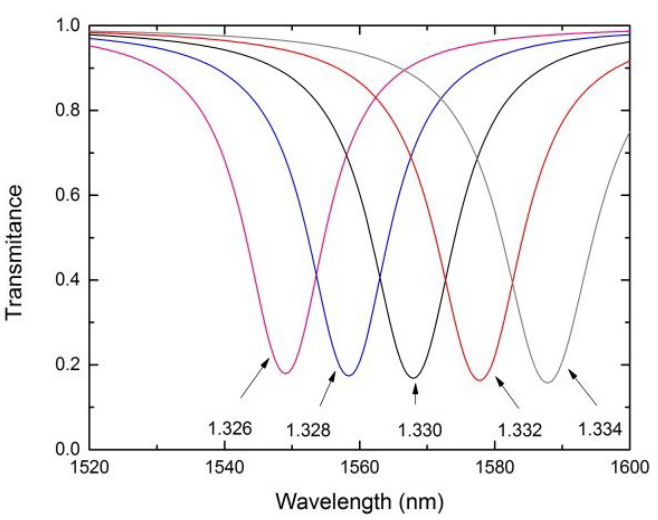

a)

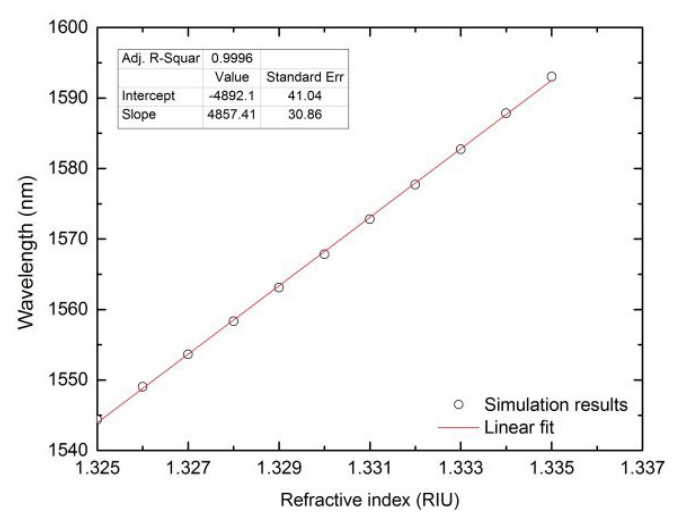

b)

Figure 2.Simulation results of the sensor: a) spectra shift with the refractive index; b) sensitivity to refractive index variations.

Figure 3-a) compares the simulated and experimental transmission spectra of the fabricated sensing head using the same parameters. A resonance with low visibility centered at $1580 \mathrm{~nm}$ is obtained, when the sensor is immersed in water with refractive index of $1.330 \mathrm{RIU}$. The position of the resonance dip is slightly shifted to lower wavelengths $(\sim 12 \mathrm{~nm})$ due to 
some inaccuracy of the different layer thicknesses. Nevertheless, the theoretical spectrum is in fairly global agreement with the experimental ones (Figure 3-b).

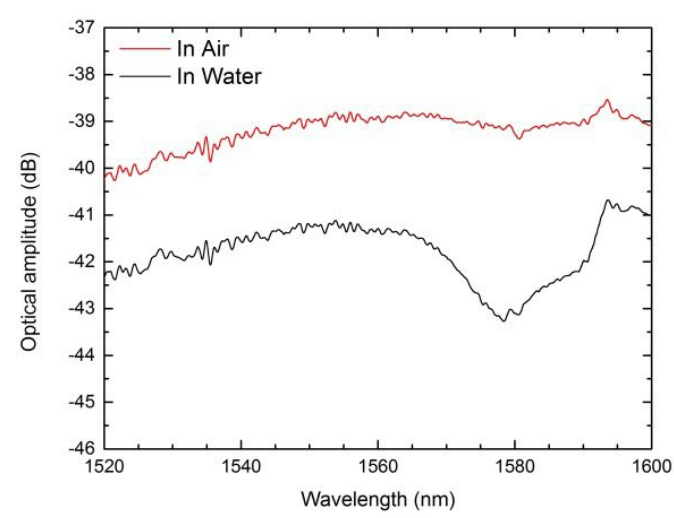

a)

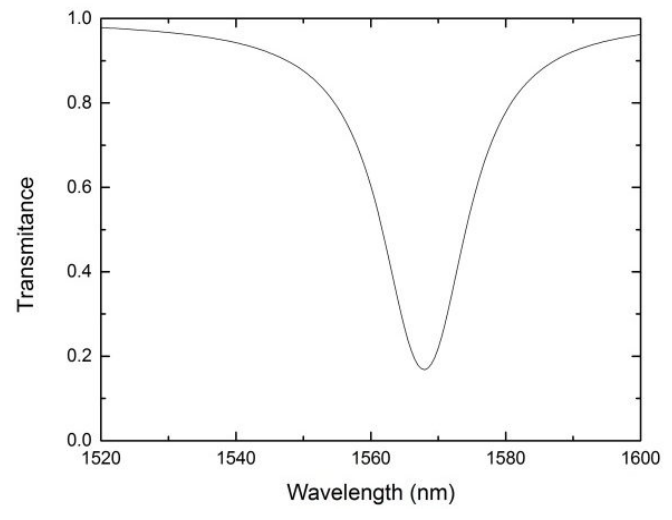

b)

Figure 3. Comparison between the experimental results (a) and numerical simulation of the sensor (b) with the same parameters.

\section{CONCLUSIONS}

A novel configuration of a surface plasmon resonance sensor in a single mode fiber was presented. The theoretical simulation preformed fits the obtained experimental results. As a further work, the fabrication of these structures will be tailored to get resonances likely the one shown in Figure 3-b and their sensing properties fully characterized considering different interrogation techniques.

\section{ACKNOLEDGMENTS}

This research was performed in the framework of project PTDC/FIS/119027/2010 (Plasmonics Based Fibre Optic Sensing with Enhanced Performance). L. Coelho would like to acknowledge FCT for providing the PhD Scholarship $\mathrm{SFRH} / \mathrm{BD} / 78149 / 2011$.

\section{REFERENCES}

[1] E. Kretschmann, and H. Raether, "Radiative decay of non radiative surface plasmons excited by light(Surface plasma waves excitation by light and decay into photons applied to nonradiative modes)," Zeitschrift Fuer Naturforschung, Teil A, 23, 2135 (1968).

[2] M. H. Chiu, "Single-mode D-type Surface Plasmon Resonance Optical Fiber Sensor: Review," Sensors \& Transducers Journal, 111(12), 18 (2009).

[3] A. Díez, M. V. Andrés, and J. L. Cruz, "In-line Fiber-optic sensors based on the excitation of surface plasma modes in metal-coated tapered Fibers," Sensors and Actuators B, 73, 4 (2001).

[4] R. Jorgenson, and S. Yee, "A fiber-optic chemical sensor based on surface plasmon resonance," Sensors and Actuators B: Chemical, 12(3), 213-220 (1993).

[5] A. K. Sharma, R. Jha, and B. Gupta, "Fiber-optic sensors based on surface plasmon resonance: a comprehensive review," Sensors Journal, IEEE, 7(8), 1118-1129 (2007).

[6] R. Slavík, J. Homola, and J. Ctyroký, "Miniaturization of fiber optic surface plasmon resonance sensor," Sensors and Actuators B, 51, 4 (1998). 
[7] J. Homola, S. S. Yee, and G. Gauglitz, "Surface plasmon resonance sensors: review," Sensors and Actuators B: Chemical, 54(1-2), 3-15 (1999).

[8] M. Kanso, S. Cuenot, and G. Louarn, "Sensitivity of optical fiber sensor based on surface plasmon resonance: modeling and experiments," Plasmonics, 3(2), 49-57 (2008).

[9] E. D. Palik, [Handbook of optical constants of solids] Academic press, (1998). 